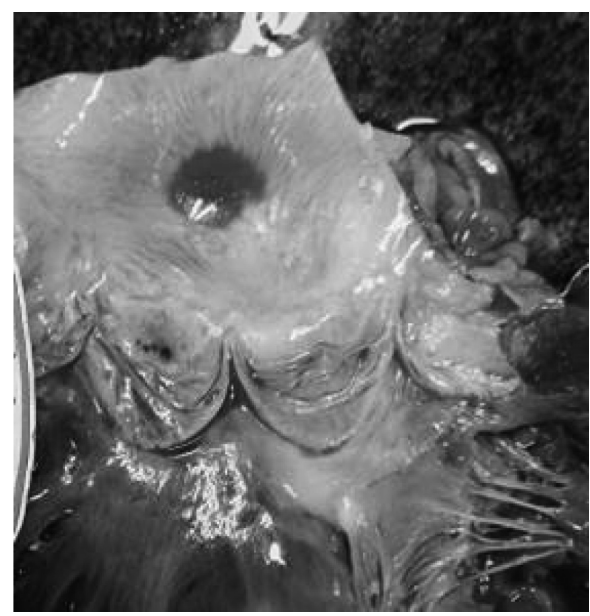

Abstract 213 Figure 2 Multiple Orifices

implies that presence of third coronary artery is a boon to the individual and the person who has third coronary artery is less likely to die from sudden natural death due to cardiac origin.

\section{A MULTISCALE HAEMORHEOLOGICAL COMPUTER- BASED MODEL OF ATHEROSCLEROSIS: AN IN-DEPTH INVESTIGATION OF ERYTHROCYTES-DRIVEN FLOW CHARACTERISTICS}

Glaucia da Conceicao Pereira. Imperial College London

\subsection{6/heartjnl-2016-309890.214}

Introduction and motivation According to the literature, the annual deaths caused by cardiovascular diseases are dramatically increasing. ${ }^{1,2}$ Atherosclerosis is a cardiovascular disease characterised by increasing arterial inflammation, which causes the blockage of the arterial lumen and the reduction of blood supply to important parts of the human body. This process is driven by fluid-mechanical forces. ${ }^{3}$ Therefore, understanding variations in blood flow characteristics driven by the erythrocytes might result in new insights on atheroma development. Hence, in this work, we present an in-deep study of haemorheological blood flow, under disease condition. Formerly, we presented a model of blood-erythrocytes in large arteries, ${ }^{3}$ comprising an established multiphase technique., ${ }^{4,5}$ This was coupled with a new fluid-structure interaction (FSI) methodology, ${ }^{3,6,7}$ which is based on immersed boundary techniques. ${ }^{8}$ Prior research reported on changes in flow due to plasmaerythrocytes interactions. ${ }^{9}$ This leads to non-Newtonian effects that are here addressed considering the effects of growing haematocrit. ${ }^{3,10,11}$ Methodology: The interaction between blood and the artery is simulated using an immersed boundary-based technique. ${ }^{3,7}$ The plasma is modelled as a Newtonian incompressible fluid using the Navier-Stokes equations. ${ }^{3,7}$ The erythrocytes are modelled as spherical particles. ${ }^{3}$ Moreover, forces resulting from the plasma-erythrocytes interactions are considered to be elastic and based on Hooke's law (DEM model of collisions), which is attained by using a multiphase flow methodology. ${ }^{4}$

Results Simulations of a single-phase fluid-structure interaction (FSI) model that mimic the coupling blood-artery have yielded results comparable with the literature. ${ }^{3,6,10,11}$ This was important to assess both the integrity and the capability of the methodology. To analyse how the erythrocytes modulate flow, we have considered a series of volume fractions $(0.00,0.15$, 0.30 , and 0.45 ) - hematocrit. We observed that, while differences in the distribution of velocities increase with increasing hematocrit, flow recirculation decreases proportionally. This indicates that the inclusion of erythrocytes might result in flow laminarization. The variations in the flow characteristics are more prominent at predilection sites for plaque deposition. We infer this might result from a natural enhancement of flow features, due to the arterial geometry at those locations.

\section{REFERENCES}

1 S. Mendis et al. WHO 2011

2 BHF CPANCDP Annual Statistics. 2014

3 G. Pereira. Imperial College London, Thesis submitted 2015

4 C. Crowe et al. CRC Press 1998

5 Y. Tsuji et al. Powder Tech. 1992

6 G. Pereira. Biophy J. 2013

7 G. Pereira et al. SIAM MMS under review 2015

8 C.S. Peskin. J. Comput. Phys. 1972

9 J. Jung et al. J. Biomech. 2006

10 G. Pereira et al. BAS. 2014

11 G. Pereira et al. BAS/BSCR 2015

12 Zhao et al. J. Comput. Phy. 2011

\section{TRANSCUTANEOUS VAGUS NERVE STIMULATION (TVNS) DECREASES SYMPATHETIC NERVE ACTIVITY IN OLDER HEALTHY HUMAN SUBJECTS}

${ }^{1}$ Aaron Murray*, ${ }^{2}$ Jennifer Clancy, ${ }^{1}$ Susan Deuchars, ${ }^{1}$ Jim Deuchars. 'University of Leeds; ${ }^{2}$ University of Glasgow; *Presenting Author

10.1136/heartjnl-2016-309890.215

Ageing is associated with autonomic dysfunction and increased risk of chronic cardiovascular disease. The aim of the present study is to assess the effects of a non-invasive method of vagus nerve stimulation, transcutaneous vagus nerve stimulation (tVNS) on autonomic activity in older healthy human subjects aged $>60$ years.

tVNS was applied to the tragus of both ears in 18 subjects $(\mathrm{F}=12, \mathrm{M}=6)$. Subjects received either $15 \mathrm{~min}$ active tVNS or sham stimulation at two visits while heart rate, blood pressure and respiration were monitored. Heart rate variability was derived from power spectral analysis of beat-to-beat oscillations in heart rate. The LF/HF ratio was calculated using low frequency power and high frequency power. Single-unit muscle sympathetic nerve activity (MSNA) was recorded using microneurography.

12 subjects $(M=6)$ had a significant decrease in $\mathrm{LF} / \mathrm{HF}$ ratio during active tVNS $(1.99 \pm 0.24$ to $1.25 \pm 0.14 ; p=$ 0.01). The remaining subjects $(\mathrm{F}=6)$ did not experience any changes in LF/HF. Male subjects had a higher baseline LF/HF $(\mathrm{M}=2.00 \pm 0.16, \mathrm{~F}=1.47 \pm 0.31 ; p=0.01)$. Low frequency power continued to decrease after active tVNS, with no change in high frequency power. No changes in $\mathrm{LF} / \mathrm{HF}$ were observed as a result of sham stimulation. Microneurography in 5 subjects detected a decrease in single-unit MSNA frequency and incidence during active tVNS $(Z=-2.02 ; p=0.04)$.

These results indicate that tVNS reduces MSNA and shifts cardiac autonomic control toward parasympathetic predominance in older adults. Further studies are needed to determine the long-term autonomic effects of tVNS in older subjects. 